\title{
Multi-modal MRI analysis with disease-specific spatial filtering: initial testing to predict mild cognitive impairment patients who convert to Alzheimer's disease
}

\author{
Kenichi Oishi ${ }^{1}{ }^{*}, K_{a z i}$ Akhter ${ }^{1}$, Michelle Mielke ${ }^{2}$, Can Ceritoglu ${ }^{3}$, Jiangyang Zhang ${ }^{1}$, Hangyi Jiang ${ }^{14}{ }^{4}, X_{i n}$ Li $^{1}$, \\ Laurent Younes ${ }^{3}$, Michael I. Miller ${ }^{3}$, Peter C. M. van Zijl ${ }^{4}$, Marilyn Albert ${ }^{5}$, Constantine G. Lyketsos ${ }^{2}$ and \\ Susumu Mori ${ }^{1,4}$
}

${ }^{1}$ Department of Radiology, Johns Hopkins University, Baltimore, MD, USA

2 Department of Psychiatry, Johns Hopkins University, Baltimore, MD, USA

${ }^{3}$ Center for Imaging Science, Johns Hopkins University, Baltimore, MD, USA

${ }^{4}$ F.M. Kirby Research Center for Functional Brain Imaging, Kennedy Krieger Institute, Baltimore, MD, USA

${ }^{5}$ Department of Neurology, Johns Hopkins University, Baltimore, MD, USA

\section{Edited by:}

Cristian Lasagna Reeves, University

of Texas Medical Branch, USA

Reviewed by:

Stefano F. Cappa, Vita-Salute San

Raffaele University, Italy

Diana Laura Castillo-Carranza,

University of Texas Medical Branch

USA

\section{*Correspondence:}

Kenichi Oishi, Department of

Radiology, Johns Hopkins University

School of Medicine, 217 Traylor

Building, 720 Rutland Avenue,

Baltimore, MD 21205, USA.

e-mail: koishi@mri.jhu.edu
Background: Alterations of the gray and white matter have been identified in Alzheimer's disease (AD) by structural magnetic resonance imaging (MRI) and diffusion tensor imaging (DTI). However, whether the combination of these modalities could increase the diagnostic performance is unknown. Methods: Participants included 19 AD patients, 22 amnestic mild cognitive impairment (aMCl) patients, and 22 cognitively normal elderly $(\mathrm{NC})$. The $\mathrm{aMCl}$ group was further divided into an "aMCl-converter" group (converted to AD dementia within 3 years), and an "aMCl-stable" group who did not convert in this time period. A $T_{1}$ weighted image, $\mathrm{aT}_{2}$ map, and a DTI of each participant were normalized, and voxel-based comparisons between $A D$ and NC groups were performed. Regions-of-interest, which defined the areas with significant differences between AD and NC, were created for each modality and named "disease-specific spatial filters" (DSF). Linear discriminant analysis was used to optimize the combination of multiple MRI measurements extracted by DSF to effectively differentiate AD from NC. The resultant DSF and the discriminant function were applied to the $\mathrm{aMCl}$ group to investigate the power to differentiate the aMCl-converters from the aMCl-stable patients. Results: The multi-modal approach with $\mathrm{AD}$-specific filters led to a predictive model with an area under the receiver operating characteristic curve (AUC) of 0.93, in differentiating aMCl-converters from aMCl-stable patients. This AUC was better than that of a single-contrast-based approach, such as $T_{1}$-based morphometry or diffusion anisotropy analysis. Conclusion: The multi-modal approach has the potential to increase the value of $\mathrm{MRI}$ in predicting conversion from $\mathrm{aMCl}$ to $\mathrm{AD}$.

Keywords: Alzheimer's disease, pre-dementia phase, mild cognitive impairment, white matter, magnetic resonance imaging, diffusion tensor imaging, multi-modal disease-specific spatial filtering

\section{INTRODUCTION}

Alzheimer's disease $(\mathrm{AD})$ is the most common cause of dementia. The importance of medical intervention in pre-dementia (Albert et al., 2011) or in the pre-symptomatic (Sperling et al., 2011) phase of $\mathrm{AD}$ has been a target of active research. For disease-preventive clinical trials, in which pre-diagnostic intervention is required, biomarkers that are linked to pathology are needed, both for early diagnosis and for monitoring, since pathological changes in $\mathrm{AD}$ are believed to have already developed prior to the onset of symptoms (Jack et al., 2010).

Efforts have been made to establish biomarkers using magnetic resonance imaging (MRI), which is a widely prevalent, non-invasive modality. A wide array of studies have characterized the structural MRI features (for review, McEvoy and Brewer, 2010) and the diffusion tensor imaging (DTI) features (for review, Chua et al., 2008; Stebbins and Murphy, 2009), related to AD.
Although robust patterns of MR-detectable pathology, such as volume loss in the medial temporal lobe or a decrease in the diffusion anisotropy of the limbic white matter (WM) fibers, have been identified, improvements in the accuracy with which $\mathrm{AD}$ can be identified is required; namely, there is a considerable amount of overlap between cognitively normal elderly participants (NC) and $\mathrm{AD}$ patients, especially in the pre-dementia phase.

To increase the accuracy of MRI in differentiating $\mathrm{AD}$ from NC, a multi-modal MRI analysis (structural MRI + DTI-derived contrasts) is one of the common approaches (Di Paola et al., 2010; Gold et al., 2010). However, whether the combination can increase the accuracy to predict conversion from MCI to AD, compared to the single-modal approach, still remains to be elucidated. In this study, we introduce a method called "multi-modal MRI analysis with disease-specific spatial filtering" (MDSF), based on the following hypotheses. First, assuming that there are multiple 
pathologies with different spatial distributions in a single disease, the optimized combination of multiple MR modalities could increase the power to separate diseased brains from normal brains. Second, if the purpose of the analysis is to investigate the existence of known pathological features of a particular disease, such features can be sensitively detected by defining regions-of-interest (ROI) that follows the expected distribution of the pathology. In MDSF, such ROIs [disease-specific spatial filters (DSF)] were created independently for multiple MR modalities (e.g., structural MRI and DTI-derived contrasts), and the MR measures extracted from the corresponding DSF were optimally combined to separate diseased brains from control brains.

In this study, images from $19 \mathrm{AD}$ patients and $22 \mathrm{NC}$ participants were used as the training dataset to create DSF for each MR contrast, followed by a linear discriminant analysis (LDA) to optimally combine the MR measures to calculate a discriminant score. We applied the resultant DSF to the test dataset, which consists of images from patients with amnestic mild cognitive impairment (aMCI), and investigated whether the resultant discriminant score could predict the conversion to AD. The classification accuracy was compared with that achieved by single MR measures or with cognitive scores.

\section{MATERIALS AND METHODS PARTICIPANTS}

We used data from a study of a well-characterized group of individuals (Mielke et al., 2009). Briefly, the study sample comprised 25 probable-AD patients (mean age, 75.6) who met NINCDS/ADRDA criteria (McKhann et al., 1984), with a clinical dementia rating (CDR) of 1; 25 aMCI patients (mean age, 75.8) who met the criteria for amnestic MCI (Petersen, 2004) with a $\mathrm{CDR}=0.5$; and $25 \mathrm{NC}$ participants (mean age, 74.3 ) with a $\mathrm{CDR}=0$. There were no differences among these groups with regard to age, race, education, and the occurrence of vascular conditions (Mielke et al., 2009). Written, informed consent was obtained under the oversight of the Johns Hopkins Institutional Review Board. Three aMCI patients and three NC participants were excluded because the DTI did not cover the whole-brain. After 3 years of follow-up, six aMCI patients had converted to AD and were defined as aMCI-converters. The remaining 16 patients were defined as aMCI-stable. Therefore, the final images used in this analyses were from 19 AD, 6 aMCI-converters, 16 aMCI-stable patients, and 22 NC participants.

\section{CLINICAL EVALUATIONS}

The diagnosis and neuropsychiatric evaluations [CDR, the Alzheimer's Disease Assessment Scale - cognitive portion (ADAS$\operatorname{cog}$ ), the mini mental state examination (MMSE), and the geriatric depression scale (GDS)] were performed at the time of the MRI scan by the Alzheimer's Disease Research Center (ADRC) staff. Re-evaluation of the diagnosis was continued annually for 3 years.

\section{MRI SCANS AND IMAGE PROCESSING}

For each participant, a DTI, co-registered, double-echo fast spin echo (DE-FSE), and a $\mathrm{T}_{1}$-weighted image were acquired using a $3 \mathrm{~T}$ scanner (Gyroscan NT, Philips Medical Systems). The parameters were as follows. DTI: single-shot echo-planar imaging; 30 diffusion weighting orientations; $b$-value $700 \mathrm{~s} / \mathrm{mm}^{2}$; 50-60 gapless wholebrain axial sections of $2.2 \mathrm{~mm}$ thickness; matrix $96 \times 96$; field of view (FOV) $212 \mathrm{~mm} \times 212 \mathrm{~mm}$; zero-filled to $256 \mathrm{~mm} \times 256 \mathrm{~mm}$. DE-FSE: first echo time (TE) $10.1 \mathrm{~ms}$; second TE $96.0 \mathrm{~ms}$; repetition time (TR) 3,000 ms; 48 gapless whole-brain axial slices of $3 \mathrm{~mm}$ thickness; matrix $256 \times 247$; FOV $240 \mathrm{~mm} \times 210 \mathrm{~mm}$; zerofilled to $256 \mathrm{~mm} \times 256 \mathrm{~mm}$. $\mathrm{T}_{1}$-weighted image: magnetizationprepared rapid gradient recalled echo; TE $3.2 \mathrm{~ms}$; TR $6.9 \mathrm{~ms}$; matrix $256 \times 256 \times 170$; FOV $240 \mathrm{~mm} \times 240 \mathrm{~mm} \times 204 \mathrm{~mm}$; zero-filled to $256 \mathrm{~mm} \times 256 \mathrm{~mm} \times 204 \mathrm{~mm}$. After the raw diffusion-weighted images were corrected for motion, eddy current, and B0-susceptibility distortion (Huang et al., 2008), a tensor field was calculated. $\mathrm{A}_{2}$ map was calculated from the DE-FSE, using the simple mono-exponential model. All images were co-registered and re-sliced to $1 \mathrm{~mm}$ isotropic resolution $(181 \times 217 \times 181$ matrix $)$.

\section{NORMALIZATION OF THE IMAGES TO THE JHU-MNI ATLAS}

Diffusion tensor imaging and $\mathrm{T}_{2}$ maps were normalized to a multi-modal JHU-MNI atlas, as previously described (Oishi et al., 2009). Briefly, DTI was transformed using first affine transformation and then large deformation diffeomorphic metric mapping (LDDMM). The resultant matrices were applied to the corresponding $\mathrm{T}_{2}$ maps for the normalization. From the normalized tensor, FA, MD, axial diffusion $\left(\lambda_{\|}\right)$, and radial diffusion $\left(\lambda_{\perp}\right)$ maps were calculated. The Jacobian map was calculated from the transformation matrix of LDDMM (called Jacobian-DTI hereafter) for the morphometric analysis.

For the analysis of the $\mathrm{T}_{1}$-weighted images, we followed a voxelbased morphometry method (Ashburner and Friston, 2000), with several modifications. First, tissue segmentation was performed for individual $\mathrm{T}_{1}$-weighted images using VBM5.1 ${ }^{1}$. A gray matter (GM) template was created from the JHU-MNI atlas the same way. There was no isotropic smoothing after segmentation. GM segmentation of each individual was normalized to the GM template, first using 12-parameter affine transformation and then using LDDMM. The Jacobian map (called Jacobian- $\mathrm{T}_{1}$ hereafter) was calculated from the transformation matrix of LDDMM.

The final products for each participant after normalizations were: Jacobian- $\mathrm{T}_{1}$; Jacobian-DTI; FA; $\mathrm{MD} ; \lambda_{\|} ; \lambda_{\perp}$; and $\mathrm{T}_{2}$ measures, mapped on the atlas space (Figure 1A). All image transformation was achieved with the software DiffeoMap ${ }^{2}$.

\section{IDENTIFICATION OF THE AREAS WITH AD-RELATED ALTERATIONS USING A TRAINING DATASET}

We performed a voxel-based comparison between $19 \mathrm{AD}$ and 22 NC patients for each MR measure, using the SPM5 software ${ }^{3}$, implemented in Matlab 6.5 (The MathWorks, Natick, MA, USA; Figure 1B). Since all images were already normalized to the atlas space (JHU-MNI atlas) by LDDMM, we used only a two-sample $t$-test of SPM5 without smoothing. The reason we did not use isotropic smoothing is that it introduces partial volume effects,

\footnotetext{
${ }^{1}$ http://dbm.neuro.uni-jena.de/vbm/

${ }^{2}$ www.MriStudio.org

${ }^{3}$ http://www.fil.ion.ucl.ac.uk/spm/
} 


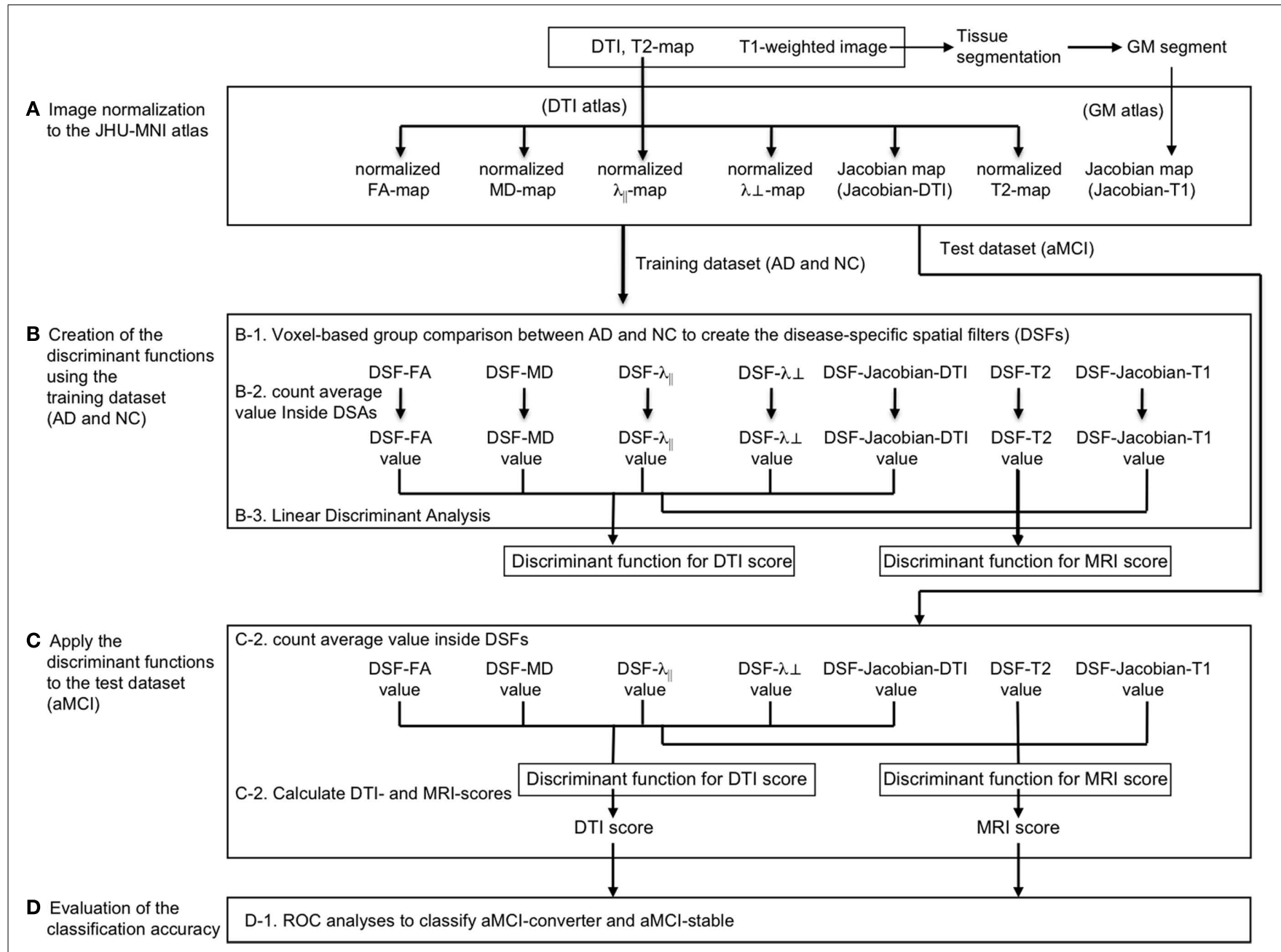

FIGURE 1 | Flow chart of the study.

especially for the WM, in which tracts with very different connectivity are adjacent to each other. The areas with statistical significance ( $p<0.05$ after correction for multiple comparisons using a false discovery rate) were binarized to create a mask called a DSF, which represented the spatial distribution of AD-related alterations in each MR parameter. The DSF of each MR parameter was called DSF-"parameter name". For example, the DSF of the FA map was called the DSF-FA. Note that the DSF-Jacobian-ex-DTI indicated areas of expansion, and the DSF-Jacobian-DTI indicated areas of atrophy.

\section{OPTIMIZATION OF THE COMBINATION OF MR MEASURES TO SEPARATE AD FROM NC USING A TRAINING DATASET}

The eight DSFs were applied to the normalized images of each subject. MR measures inside the corresponding DSF were averaged and called the DSF-"parameter name" value. As a result, each subject had eight DSF values.

In order to combine the DSF values to increase the classification power, we used LDA. Separate LDFs were performed using the DTI-derived values (Jacobian-DTI, Jacobian-ex-DTI, FA, MD, $\lambda_{\|}$, and $\lambda_{\perp}$ ), and all the MR values (Jacobian- $T_{1}$, Jacobian-DTI, Jacobian-ex-DTI, FA, MD, $\lambda_{\|}, \lambda_{\perp}$, and $\mathrm{T}_{2}$ ), as the input variables.
The classification score for DTI-derived DSF values was called the DTI score, and the classification score for all DSF values was called the MRI score.

\section{APPLICATION OF THE OPTIMIZED COMBINATION OF DSF TO THE AMCI GROUP}

Previous steps included the creation of the DSFs and discriminant function to calculate discriminant scores (DTI score and MRI score), which were required to perform MDSF. We applied the MDSF to the aMCI group (Figure 1C) to see whether the DTI score and the MRI score could accurately classify aMCI-converters and aMCI-stable patients (Figure 1D). The ROC analyses were performed to assess the classification performance. SPSS 19 (IBM Corporation, New York, USA) was used to create ROC curves. MedCalc 11.5.1 (MedCalc Software, Mariakerke, Belgium) was used for the non-parametric pair-wise comparison between the areas under the ROC curves.

\section{COMPARISON BETWEEN MRI SCORE AND COGNITIVE SCALES}

We performed permutation tests and ROC analysis for the two types of cognitive scales (ADAS-cog and MMSE), as well as for the number of correct answers in the immediate and delayed story 
recall of the WMS-III, in order to assess the accuracy of separating aMCI-converter from aMCI-stable patients.

\section{RESULTS}

\section{NORMALIZATION OF THE IMAGES TO THE JHU-MNI ATLAS}

All 63 images were normalized to the JHU-MNI template. In Figure 2, the averaged FA map (first to fifth rows), the averaged $\mathrm{T}_{2}$ map (sixth row), and the averaged GM segmentation (seventh row) are shown. The extent of the anatomical details appreciated in these figures represents the accuracy of the normalization.

\section{CREATION OF DSFs}

The areas with statistical significance that differentiated AD patients from NC subjects were overlaid on the averaged images (Figure 2). The WM alterations were mainly found in the limbic fibers (the fornix, the cingulum hippocampal area, and the posterior cingulate), the forceps major and minor (including the splenium and the genu of the corpus callosum), and the periventricular WM areas. In addition, small areas with significant alterations were scattered in the gyrus rectus, the inferior frontal gyrus, and the temporal-parietal lobes. Generally, the limbic fibers and the forceps showed a decrease in the FA, with an increase in the MD, which was mainly caused by a greater increase in the $\lambda_{\perp}$ than in the $\lambda_{\|}$. Among these fibers, the fornix had the most striking change, with atrophy and increased $\mathrm{T}_{2}$. On the other hand, a general increase in diffusivity, accompanied by a $\mathrm{T}_{2}$ increase, was found in the area surrounding the lateral ventricles. To eliminate the possibility that the decreased FA and increased diffusivity were due to misregistration of the ventricle, the ventricles of each image were manually defined and normalized by applying the corresponding transformations. Misregistration of the ventricle was not found in any of the abnormal FA/diffusivity areas, except for

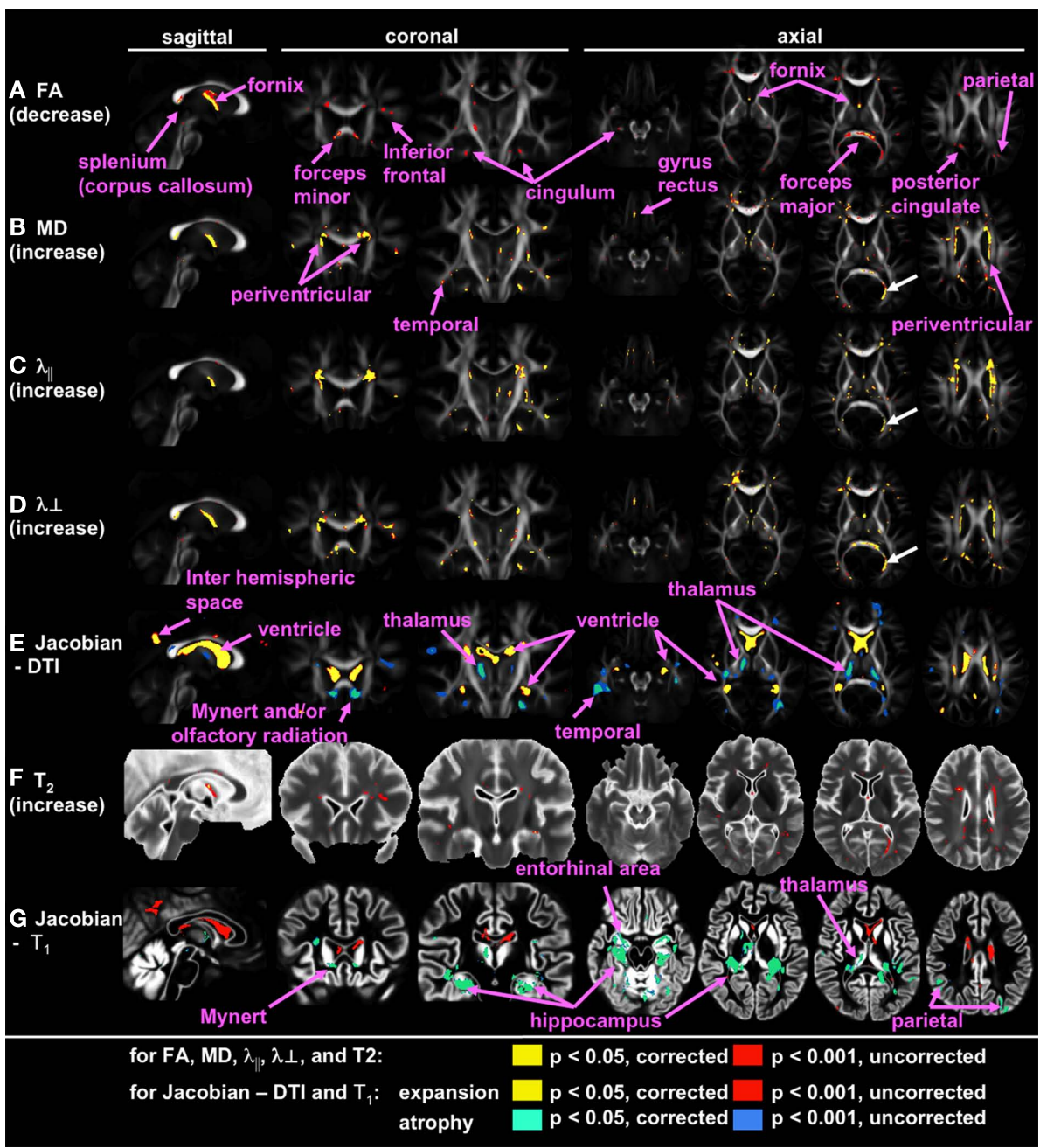

FIGURE 2 | Voxel-based group comparison between AD and NC. Areas with signal or volume alterations in $A D$ compared to $N C$ are shown as colored maps, overlaid on an averaged FA map (A-E), an averaged $T_{2}$ map (F), and an averaged GM segmentation map (G), created from all 63 images. (A): Areas with reduced FA. (B) Areas with increased MD. (C) Areas with increased $\lambda_{\|}$. (D) Areas with increased $\lambda_{\perp}$. (E) Areas with an increased and decreased Jacobian, which was calculated from a transformation matrix obtained from the normalization of DTI. (F) Area with increased $T_{2}$. (G) Areas with increased and decreased Jacobian, which were calculated from a transformation matrix obtained from the normalization of a GM segmentation map. White arrows show the misregistration seen in the left posterior horn of the lateral ventricle. 
the area medial to the left posterior horn of the lateral ventricle, indicated by the white arrow in Figure 2. The significant changes in FA and diffusivity in this area, therefore, were artifacts.

Gray matter atrophy was mainly found in the hippocampus, the entorhinal area, and the thalamus. Small areas with significant atrophy were also found in the parietal cortices. Atrophy was also found by both Jacobian-DTI and Jacobian- $\mathrm{T}_{1}$ in the area where the basal nucleus of Meynert and the olfactory radiation are located.

The areas with significant $\mathrm{AD}$-related alterations in each $\mathrm{MR}$ measure were binarized to create DSFs for $\mathrm{AD}$, as shown in Figure 3. DSA-values for each MR measure were calculated and are demonstrated in Figure 4.

\section{OPTIMIZATION OF THE COMBINATION OF DISEASE-SPECIFIC ATLASES}

Linear discriminant analysis provided discriminant functions to calculate a DTI score and an MRI score. The structure coefficients for each DSF value to calculate a DTI score were: DSFJacobian-DTI value, 0.726; DSF-Jacobian-ex-DTI value, -0.502 ; DSF-FA value, 0.878 ; DSF-MD value, -0.785 ; DSF- $\lambda_{\|}$value, -692 ; and DSF- $\lambda_{\perp}$ value, -0.813 . The structure coefficients for each DSF value to calculate an MRI score were: DSF-Jacobian- $T_{1}$ value, 0.460; DSF-Jacobian-DTI value, 0.722; DSF-Jacobian-exDTI value, -0.499 ; DSF-FA value, 0.876; DSF-MD value, -0.781 ; DSF- $\lambda_{\|}$value, -0.689 ; DSF- $\lambda_{\perp}$ value, -0.809 ; and DSF-T 2 value, -0.687 .

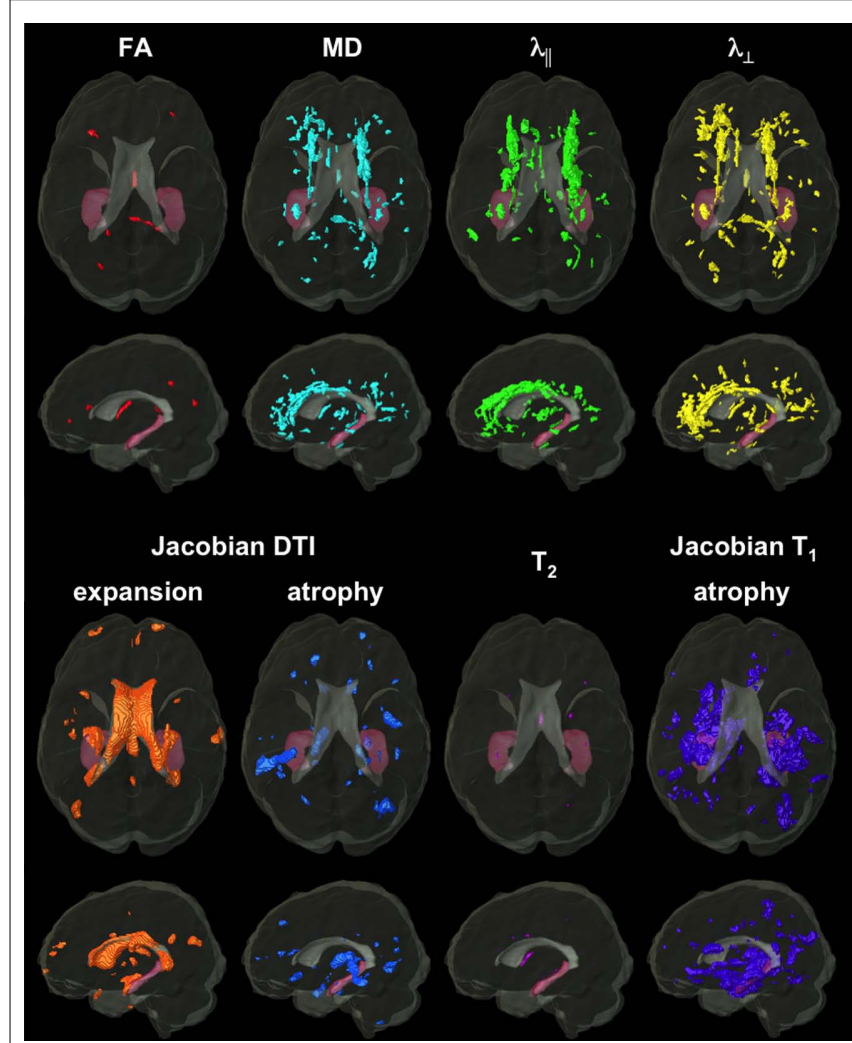

FIGURE 3 |Top and left-side view of the eight disease-specific spatial filters (DSFs) created from voxel-based statistical comparisons of the training dataset (AD and $\mathbf{N C}$ ). The brain surface and the hippocampal surface of the JHU-MNI atlas are shown in gray and pink, respectively.

\section{APPLICATION OF THE MDSF TO THE MCI GROUP}

We applied the MDSF (combination of the DSF and discriminant function from the training dataset) to an aMCI group as a test dataset, to calculate a DTI score and an MRI score of each patient (Figure 4). The ROC curves, the AUCs, and the classification functions based on the optimal cutoffs, are shown in Figure 5A and Table 1. Pair-wise comparison of the ROC curves demonstrated that the MRI score was significantly better than any scores with the single contrast approach, although the difference between MRI score and DTI score was not significant.

\section{COMPARISON BETWEEN MRI SCORE AND COGNITIVE SCALES}

Among the selected cognitive tests, the number of correct answers in the delayed story recall of the WMS-III could best predict the conversion from $\mathrm{MCI}$ to $\mathrm{AD}$, with an AUC of 0.83 , which tended to be less than that $(0.93)$ of the MRI score, even though the difference did not reach the statistical significance (Figure 5B; Table 1).

\section{DISCUSSION}

\section{SPATIAL SPECIFICITY OF THE WM AND GM ALTERATIONS}

We found two different patterns of WM alterations in AD. Namely, an FA reduction associated with a $\lambda_{\perp}$-predominant increase in diffusivity in the limbic fibers and the forceps, and a general increase in diffusivity with a $\mathrm{T}_{2}$ increase found in the periventricular area. Alterations in the fornix, cingulum, and the splenium of the corpus callosum were consistent with earlier ROI studies (Naggara et al., 2006; Ringman et al., 2007; Zhang et al., 2007; Mielke et al., 2009). A recent study using tract-based analysis (TBSS; Smith et al., 2006), also detected alterations in these structures (Damoiseaux et al., 2009; Acosta-Cabronero et al., 2010), supporting the validity of our findings. A test for CSF contamination suggested a highly accurate registration by LDDMM, with a minimal CSF contribution. An exception was in the areas around the posterior horn of the lateral ventricles, which were eliminated from the subsequent analyses. The periventricular WM alterations in $\mathrm{AD}$ are in accordance with past findings, where they were designated periventricular hyperintense lesions (PVH; Targosz-Gajniak et al., 2009). We also identified alterations in the superficial WM (Oishi et al., 2008) that had been found in past studies (Bozzali et al., 2002; Damoiseaux et al., 2009; Zhang et al., 2009).

Our main findings with regard to GM morphometry in AD mostly agree with past findings, showing atrophy in the hippocampal complex, the basal nucleus of Meynert, and the thalamus (Jack et al., 1999; Morris et al., 2002; Karas et al., 2004; Teipel et al., 2005; de Jong et al., 2008; McEvoy et al., 2009). However, the distribution of the abnormalities was limited in our study, compared to the previous studies. One of the reasons for this was that our voxel-based analysis did not use spatial smoothing, which would have led to lower sensitivity to detect abnormalities.

\section{PREDICTIVE ABILITY OF THE MDSF}

The overall agreement of the above VBA findings with previous reports confirms that our DSF capture most locations with significant anatomical alterations. Once the locations with a potential abnormality are encoded in the DSF (Figure 3), the MRI/DTI scores can be generated for each patient by applying the DSFs to the multi-modal MRI data. The ROC analyses indicated that the MRI and DTI scores better predicted conversion from aMCI to 


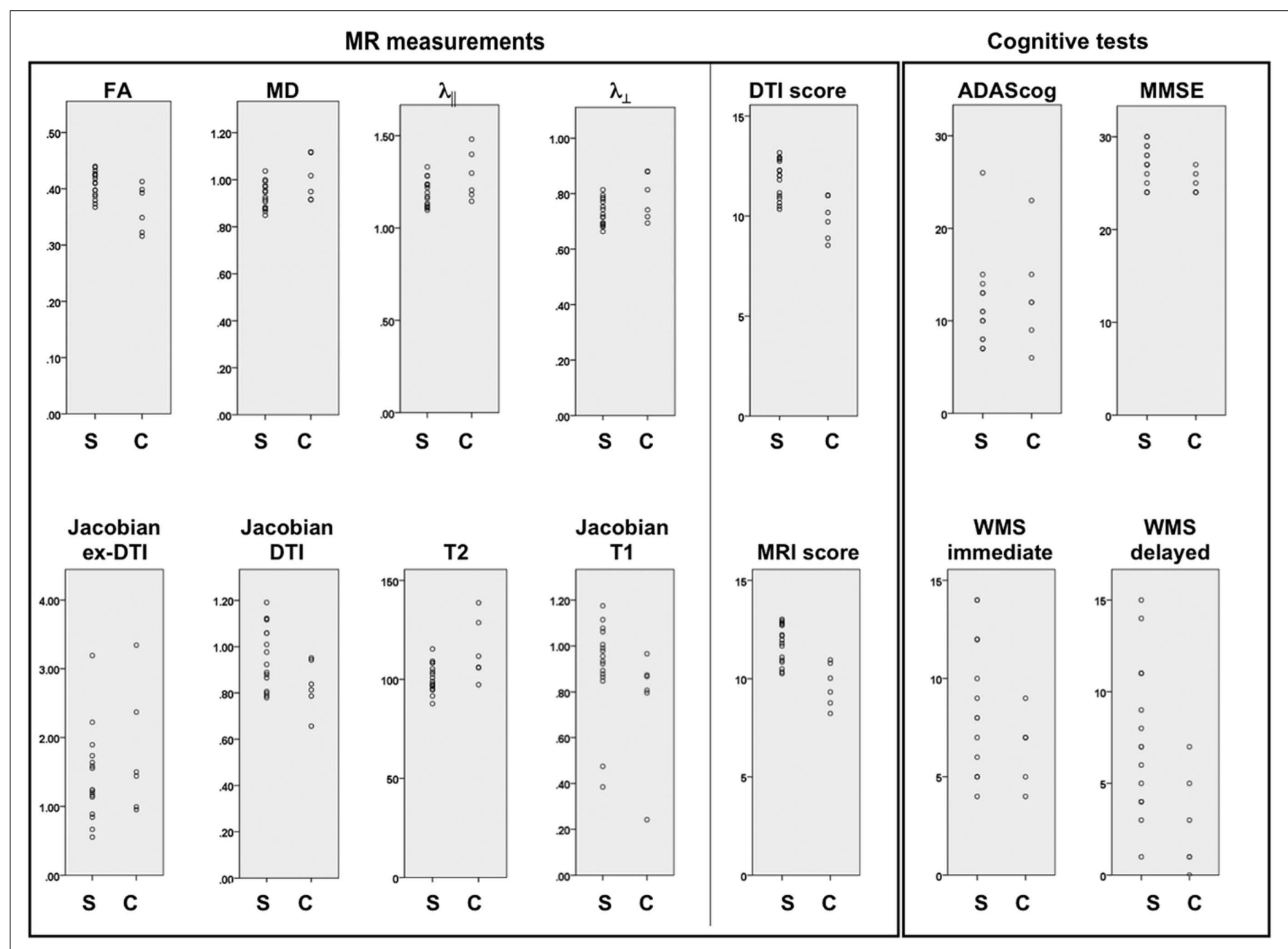

FIGURE 4 | Scattergrams of the measured DSF values of the eight parameters, the discriminant scores (DTI score and MRI score), and the results of cognitive tests of the test dataset (MCl-converter and $\mathbf{M C l}-$ stable). $\mathrm{MD}, \lambda_{\|}$, and $\lambda_{\perp}: 10^{-3} \times \mathrm{mm}^{2} / \mathrm{s} ; \mathrm{T}_{2}: \mathrm{ms} \mathrm{C}: \mathrm{aMCl}-\mathrm{converter} ; \mathrm{S}: \mathrm{aMCl}-\mathrm{stable}$.
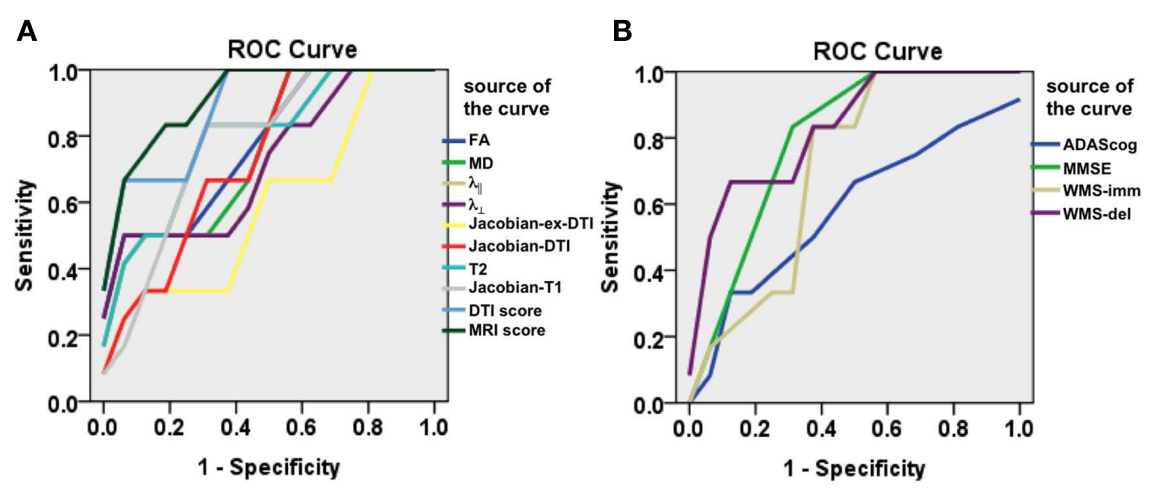

FIGURE 5 | Results of the receiver operating characteristic curve (ROC) analyses. (A) The ROCs of various MR measurements. (B) The ROCs of various cognitive tests. WMS-imm: number of correct answers in the immediate story recall of the WMS-III; WMS-del: number of correct answers in the delayed story recall of the WMS-III. 
Table 1 | Result of the ROC curve analysis to separate aMCl-converter from aMCl-stable patients.

\begin{tabular}{|c|c|c|c|c|c|c|c|}
\hline & AUC & $95 \% \mathrm{Cl}$ of $\mathrm{AUC}$ & Optimal cut-off* & Sensitivity & Specificity & vs. DTI score ( $p$ value) & vs. MRI score ( $p$ value) \\
\hline MD & 0.76 & $0.53-0.91$ & $>0.9 \mathrm{E}-03$ & 1.00 & 0.50 & 0.026 & 0.023 \\
\hline$\lambda_{\perp}$ & 0.73 & $0.50-0.89$ & $>0.8 \mathrm{E}-03$ & 0.50 & 1.00 & 0.032 & 0.030 \\
\hline Jacobian-ex-DTI & 0.59 & $0.37-0.80$ & $>1.9$ & 0.33 & 0.94 & 0.009 & 0.007 \\
\hline Jacobian- $T_{1}$ & 0.69 & $0.46-0.87$ & $<0.94$ & 0.83 & 0.69 & 0.055 & 0.034 \\
\hline DTI score & 0.91 & $0.71-0.99$ & $<0.0$ & 1.00 & 0.75 & N/A & 0.140 \\
\hline MRI score & 0.93 & $0.73-0.99$ & $<0.0$ & 1.00 & 0.75 & 0.140 & N/A \\
\hline ADAS-cog & 0.56 & $0.34-0.77$ & $>11$ & 0.67 & 0.63 & 0.033 & 0.026 \\
\hline MMSE & 0.79 & $0.57-0.94$ & $<25$ & 0.83 & 0.69 & 0.160 & 0.105 \\
\hline
\end{tabular}

Results of the pair-wise comparison of ROC curves between the DTI score and single-modality approaches (vs. DTI score), and the MRI score and single-modality approaches (vs. MRI score), are shown in the two right columns.

${ }^{*} M D, \lambda_{\|}$, and $\lambda_{\perp}: \mathrm{mm}^{2} / \mathrm{s} ; T_{2}: m s$.

$\mathrm{AD}$, compared to the analysis using a single $\mathrm{MR}$ contrast alone or cognitive scales. This indicates the potential of multi-modal measurement to increase the value of MRI in the early diagnosis of AD.

There were several reasons we think the MDSF approach could classify aMCI-converters from aMCI-stable patients better than the single-modal approach. First, multiple structures with different pathologies are involved in AD. Especially in the early phase, WM alteration detected by DTI-derived measurements has been found independently of the GM atrophy (Bai et al., 2009; Agosta et al., 2011). Therefore, diffusion measurements and morphometric measurements seems to be complementary, and the combination of these would capture the pathology of AD comprehensively. Second, AD is one of the neurodegenerative diseases in which particular brain structures systematically and gradually degenerate. This systematic feature (not random) is ideal for creating the DSF.

\section{LIMITATIONS}

The main limitations of the current study were the small number of the training dataset ( $\mathrm{AD}$ and $\mathrm{NC}$ ) and the testing dataset (aMCI). Beyond the technical demonstration, the use of larger training and

\section{REFERENCES}

Acosta-Cabronero, J., Williams, G. B., Pengas, G., and Nestor, P. J. (2010). Absolute diffusivities define the landscape of white matter degeneration in Alzheimer's disease. Brain 133, 529-539.

Agosta, F., Pievani, M., Sala, S., Geroldi, C., Galluzzi, S., Frisoni, G. B., and Filippi, M. (2011). White matter damage in Alzheimer disease and its relationship to gray matter atrophy. Radiology 258, 853-863.

Albert, M. S., Dekosky, S. T., Dickson, D., Dubois, B., Feldman, H. H., Fox, N. C., Gamst, A., Holtzman, D. M.,
Jagust, W. J., Petersen, R. C., Snyder, P. J., Carrillo, M. C., Thies, B., and Phelps, C. H. (2011). The diagnosis of mild cognitive impairment due to Alzheimer's disease: recommendations from the National Institute on Aging-Alzheimer's Association workgroups on diagnostic guidelines for Alzheimer's disease. Alzheimers Dement. 7, 270-279.

Ashburner, J., and Friston, K. J. (2000). Voxel-based morphometry-the methods. Neuroimage 11, 805-821.

Bai, F., Zhang, Z., Watson, D. R., Yu, H., Shi, Y., and Yuan, Y. (2009).

testing datasets would be necessary to create DSFs and to evaluate their usefulness in actual clinical settings. The application of this method to the diagnosis of other neurodegenerative diseases would also be necessary to further evaluate the efficacy of this method.

In summary, the MDSF approach increased the predictive power of MRI to identify aMCI patients who might convert to AD. This method has the potential to quantitatively analyze MRI data to identify patients at risk for developing AD.

\section{ACKNOWLEDGMENTS}

The authors thank Ms. Mary McAllister for help with manuscript editing. This study was supported by NIH grants, R21AG033774, P50AG005146 (Kenichi Oishi), R21 AG028754, R21NS060271-01 (Michelle Mielke), P41 RR015241, P41 RR015241, U24RR021382, PO1EB00195, and RO1AG20012 (Susumu Mori), and by the Johns Hopkins Alzheimer's Disease Research Center (Kenichi Oishi). The images acquired from Alzheimer's patients and age-matched controls were obtained with the help of a methods development grant from Glaxo-Smith-Kline, awarded to Marilyn Albert and Constantine G. Lyketsos.

Abnormal white matter independent of hippocampal atrophy in amnestic type mild cognitive impairment. Neurosci. Lett. 462, 147-151.

Bozzali, M., Falini, A., Franceschi, M., Cercignani, M., Zuffi, M., Scotti, G., Comi, G., and Filippi, M. (2002). White matter damage in Alzheimer's disease assessed in vivo using diffusion tensor magnetic resonance imaging. J. Neurol. Neurosurg. Psychiatr. 72, 742-746.

Chua, T. C., Wen, W., Slavin, M. J., and Sachdev, P. S. (2008). Diffusion tensor imaging in mild cognitive impairment and Alzheimer's disease: a review. Curr. Opin. Neurol. 21, 83-92.

Damoiseaux, J. S., Smith, S. M., Witter, M. P., Sanz-Arigita, E. J., Barkhof, F., Scheltens, P., Stam, C. J., Zarei, M., and Rombouts, S. A. (2009). White matter tract integrity in aging and Alzheimer's disease. Hum. Brain Mapp. 30, 1051-1059.

de Jong, L. W., Van Der Hiele, K., Veer, I. M., Houwing, J. J., Westendorp, R G., Bollen, E. L., De Bruin, P. W., Middelkoop, H. A., Van Buchem, M. A., and Van Der Grond, J. (2008). Strongly reduced volumes of putamen and thalamus in Alzheimer's 
disease: an MRI study. Brain 131, 3277-3285.

Di Paola, M., Di Iulio, F., Cherubini, A., Blundo, C., Casini, A. R., Sancesario, G., Passafiume, D., Caltagirone, C., and Spalletta, G. (2010). When, where, and how the corpus callosum changes in MCI and AD: a multimodal MRI study. Neurology 74, 1136-1142.

Gold, B. T., Powell, D. K., Andersen, A. H., and Smith, C. D. (2010). Alterations in multiple measures of white matter integrity in normal women at high risk for Alzheimer's disease. Neuroimage 52, 1487-1494.

Huang, H., Ceritoglu, C., Li, X., Qiu, A., Miller, M. I., Van Zijl, P. C., and Mori, S. (2008). Correction of B0 susceptibility induced distortion in diffusion-weighted images using large-deformation diffeomorphic metric mapping. Magn. Reson. Imaging 26, 1294-1302.

Jack, C. R. Jr., Bernstein, M. A., Borowski, B. J., Gunter, J. L., Fox, N. C., Thompson, P. M., Schuff, N., Krueger, G., Killiany, R. J., Decarli, C. S., Dale, A. M., Carmichael, O. W., Tosun, D., and Weiner, M. W. (2010). Update on the magnetic resonance imaging core of the Alzheimer's disease neuroimaging initiative. Alzheimers Dement. 6, 212-220.

Jack, C. R. Jr., Petersen, R. C., Xu, Y. C., O’Brien, P. C., Smith, G. E., Ivnik, R. J., Boeve, B. F., Waring, S. C., Tangalos, E. G., and Kokmen, E. (1999). Prediction of AD with MRIbased hippocampal volume in mild cognitive impairment. Neurology 52, 1397-1403.

Karas, G. B., Scheltens, P., Rombouts, S. A., Visser, P. J., Van Schijndel, R. A., Fox, N. C., and Barkhof, F. (2004). Global and local gray matter loss in mild cognitive impairment and Alzheimer's disease. Neuroimage 23, 708-716.

McEvoy, L. K., and Brewer, J. B. (2010). Quantitative structural MRI for early detection of Alzheimer's disease. Expert Rev. Neurother. 10, 1675-1688.
McEvoy, L. K., Fennema-Notestine, C., Roddey, J. C., Hagler, D. J. Jr., Holland, D., Karow, D. S., Pung, C. J., Brewer, J. B., and Dale, A. M. (2009). Alzheimer disease: quantitative structural neuroimaging for detection and prediction of clinical and structural changes in mild cognitive impairment. Radiology 251, 195-205.

McKhann, G., Drachman, D., Folstein, M., Katzman, R., Price, D., and Stadlan, E. M. (1984). Clinical diagnosis of Alzheimer's disease: report of the NINCDS-ADRDA Work Group under the auspices of Department of Health and Human Services Task Force on Alzheimer's disease. Neurology 34, 939-944.

Mielke, M. M., Kozauer, N. A., Chan, K. C., George, M., Toroney, J., Zerrate, M., Bandeen-Roche, K., Wang, M. C., Vanzijl, P., Pekar, J. J., Mori, S., Lyketsos, C. G., and Albert, M. (2009). Regionally-specific diffusion tensor imaging in mild cognitive impairment and Alzheimer's disease. Neuroimage 46, 47-55.

Morris, J. C., Csernansky, J., and Price, J. L. (2002). MRI measures of entorhinal cortex versus hippocampus in preclinical AD. Neurology 59, 1474; author reply 1474-1475.

Naggara, O., Oppenheim, C., Rieu, D., Raoux, N., Rodrigo, S., Dalla Barba, G., and Meder, J. F. (2006). Diffusion tensor imaging in early Alzheimer's disease. Psychiatry Res. 146, 243-249.

Oishi, K., Faria, A., Jiang, H., Li, X., Akhter, K., Zhang, J., Hsu, J. T., Miller, M. I., Van Zijl, P. C., Albert, M., Lyketsos, C. G., Woods, R., Toga, A. W., Pike, G. B., RosaNeto, P., Evans, A., Mazziotta, J., and Mori, S. (2009). Atlas-based whole brain white matter analysis using large deformation diffeomorphic metric mapping: application to normal elderly and Alzheimer's disease participantstlas. Neuroimage 46, 486-499.

Oishi, K., Zilles, K., Amunts, K., Faria, A., Jiang, H., Li, X., Akhter, K., Hua, K., Woods, R., Toga, A. W., Pike, G. B., Rosa-Neto, P., Evans, A., Zhang, J., Huang, H., Miller, M. I., Van Zijl,
P. C., Mazziotta, J., and Mori, S. (2008). Human brain white matter atlas: identification and assignment of common anatomical structures in superficial white matter. Neuroimage 43, 447-457.

Petersen, R. C. (2004). Mild cognitive impairment as a diagnostic entity. $J$. Intern. Med. 256, 183-194.

Ringman, J. M., O’Neill, J., Geschwind, D., Medina, L., Apostolova, L. G., Rodriguez, Y., Schaffer, B., Varpetian, A., Tseng, B., Ortiz, F., Fitten, J., Cummings, J. L., and Bartzokis, G. (2007). Diffusion tensor imaging in preclinical and presymptomatic carriers of familial Alzheimer's disease mutations. Brain 130, 1767-1776.

Smith, S. M., Jenkinson, M., JohansenBerg, H., Rueckert, D., Nichols, T. E., Mackay, C. E., Watkins, K. E., Ciccarelli, O., Cader, M. Z., Matthews, P. M., and Behrens, T. E. (2006). Tractbased spatial statistics: voxelwise analysis of multi-subject diffusion data. Neuroimage 31, 1487-1505.

Sperling, R. A., Aisen, P. S., Beckett, L. A., Bennett, D. A., Craft, S., Fagan, A. M., Iwatsubo, T., Jack, C. R. Jr., Kaye, J., Montine, T. J., Park, D. C., Reiman, E. M., Rowe, C. C., Siemers, E., Stern, Y., Yaffe, K., Carrillo, M. C., Thies, B., Morrison-Bogorad, M., Wagster, M. V., and Phelps, C. H. (2011). Toward defining the preclinical stages of Alzheimer's disease: recommendations from the National Institute on Aging-Alzheimer's Association workgroups on diagnostic guidelines for Alzheimer's disease. Alzheimers Dement. 7, 280-292.

Stebbins, G. T., and Murphy, C. M. (2009). Diffusion tensor imaging in Alzheimer's disease and mild cognitive impairment. Behav. Neurol. 21, 39-49.

Targosz-Gajniak, M., Siuda, J., Ochudlo, S., and Opala, G. (2009). Cerebral white matter lesions in patients with dementia - from MCI to severe Alzheimer's disease. J. Neurol. Sci. 283, 79-82.

Teipel, S. J., Flatz, W. H., Heinsen, H., Bokde, A. L., Schoenberg, S. O., Stockel, S., Dietrich, O., Reiser, M. F., Moller, H. J., and Hampel, H.
(2005). Measurement of basal forebrain atrophy in Alzheimer's disease using MRI. Brain 128, 2626-2644.

Zhang, Y., Schuff, N., Du, A. T., Rosen, H. J., Kramer, J. H., GornoTempini, M. L., Miller, B. L., and Weiner, M. W. (2009). White matter damage in frontotemporal dementia and Alzheimer's disease measured by diffusion MRI. Brain 132, 2579-2592.

Zhang, Y., Schuff, N., Jahng, G. H., Bayne, W., Mori, S., Schad, L., Mueller, S., Du, A. T., Kramer, J. H., Yaffe, K., Chui, H., Jagust, W. J., Miller, B. L., and Weiner, M. W. (2007). Diffusion tensor imaging of cingulum fibers in mild cognitive impairment and Alzheimer disease. Neurology 68, 13-19.

Conflict of Interest Statement: Peter C. M. van Zijl is a paid lecturer for Philips Medical Systems and is the inventor of a technology that is licensed to Philips. This arrangement has been approved by the Johns Hopkins University in accordance with its conflict of interest policies.

Received: 25 July 2011; accepted: 08 August 2011; published online: 24 August 2011.

Citation: Oishi K, Akhter K, Mielke $M$, Ceritoglu C, Zhang J, Jiang $H$, Li X, Younes L, Miller MI, van Zijl PCM, Albert M, Lyketsos CG and Mori $S$ (2011) Multi-modal MRI analysis with disease-specific spatial filtering: initial testing to predict mild cognitive impairment patients who convert to Alzheimer's disease. Front. Neur. 2:54. doi: 10.3389/fneur.2011.00054

This article was submitted to Frontiers in Dementia, a specialty of Frontiers in Neurology.

Copyright () 2011 Oishi, Akhter, Mielke, Ceritoglu, Zhang, Jiang, Li, Younes, Miller, van Zijl, Albert, Lyketsos and Mori. This is an open-access article subject to a non-exclusive license between the authors and Frontiers Media SA, which permits use, distribution and reproduction in other forums, provided the original authors and source are credited and other Frontiers conditions are complied with. 